hep-th/9705101

\title{
New Constraints on the Yukawa-Type Hypothetical Interaction From The Recent Casimir Force Measurement
}

\author{
M. Bordag】, G.T. Gillies] and V.M. Mostepanenkd
}

\begin{abstract}
We calculate the constraints on the constants of hypothetical long-range interactions which follow from the recent measurement of the Casimir force. A comparison with previous constraints is given. The new constraints are up to a factor of 3000 stronger in some parameter regions .
\end{abstract}

\section{Introduction}

The existence of Yukawa-type long-range interactions between the atoms of macrobodies is predicted by the modern unified gauge-field theories of fundamental interactions, from the principles of supersymmetry and supergravity. These forces may be understood as a result of the exchange of light elementary particles (such as the axion, scalar axion, dilaton, spin-one antigraviton etc. [1]). They may be called long-range because their range of action is much larger than the typical nuclear sizes. Additional Yukawa-type interactions may arise also from corrections to the Newtonian gravitational law at small distances [2, 3]. At present, this subject is actively investigated. There are a large number of papers from both theoreticians and experimentalists. An extended annotative collection of references can be found in [4].

The most reliable way to get constraints on the parameters of the Yukawa-type long-range interactions is via laboratory experiments which are sensitive to the presence of additional forces. Examples are the Eötvös-, Galileo- and Cavendish-type experiments, Casimir and van der Waals force measurements, and measurements of transition probabilities in exotic atoms.

A collection of modern constraints following from the above-mentioned experiments can be found in [5].

Casimir forces which arise between closely located surfaces of solid bodies due to the presence of electromagnetic vacuum fluctuations provide us with one of the most interesting possibilities to restrict the permissable strength of the hypothetical

\footnotetext{
${ }^{1}$ Institute for Theoretical Physics, Leipzig University, Augustusplatz 10/11, 04109 Leipzig, Germany, http://www.physik.uni-leipzig.de/ bordag

${ }^{2}$ Department of Physics, University of Virginia, Charlottesville, Virginia 22901, email: gtg@faraday.clas.virginia.edu

${ }^{3}$ Department of Physics, Federal University of Paraiba, C.P. 5008, CEP 58059-970, Joao Pessoa, Pb-Brazil, e-mail: mostep@dfjp.ufpb.br (on leave from A.Friedmann Laboratory for Theoretical Physics, St.Petersburg, Russia)
} 
interaction. A detailed analysis of the Casimir effect, including those aspect under discussion here can be found in the monograph [6].

It was first suggested in [7] that constraints on the Yukawa-type interactions can be derived from the Casimir effect. The strongest constraints, which follow from the Casimir force measurement between a quartz plate and a spherical lens, were obtained in [5, 8] (see also [6]). They provide the strongest constraints for a range $\lambda$ of the Yukawa forces within $10^{-8} \mathrm{~m} \leq \lambda \leq 10^{-4} \mathrm{~m}$. For $\lambda \geq 10^{-4} \mathrm{~m}$ the best constraints followed from the Cavendish- and Eötvös-type experiments [5, 8] and for $\lambda \leq 10^{-8} \mathrm{~m}$ from van der Waals force measurements and from measurements of transition probabilities in exotic atoms [9]. Therefore new, more exact Casimir force measurements are of prime importance in order to obtain stronger constraints on the constants of Yukawa-type hypothetical interactions in the intermediate range of $\lambda$.

Recently, a new experiment was performed [10], in which the Casimir force was measured between two metallised surfaces, a plane disc and a spherical lens with a distance $a$ between them. The theoretical value, obtained in [11], was confirmed within an accuracy of $5 \%$ in the distance region $0.6 \mu \mathrm{m} \leq a \leq 6 \mu \mathrm{m}$. Note that this was the first direct measurement of the Casimir force for metallic surfaces since Sparnaay [12], and that the accuracy has been increased in 20 times. The metallic surface of the bodies (a $0.5 \mu \mathrm{m}$ thick gold layer) increased considerably the contribution of the hypothetical Yukawa forces due to the high density of gold (the Yukawa forces are proportional to the density squared). Also, the relatively large spacing between the bodies, up to $6 \mu \mathrm{m}$, where the Casimir force is very small, turned out to be essential for the strengthening of the constraints.

In the present paper new constraints on parameters of the Yukawa-type hypothetical interactions, which follow from the experiment [10] on Casimir force measurement, are obtained. They turn out to be stronger than those obtained previously from the measurement of the Casimir force between dielectrics and from one of the versions of a Cavendish-type experiment in the range $6.310^{-8} \mathrm{~m} \leq \lambda \leq 3.110^{-3} \mathrm{~m}$. Especially within $210^{-6} \mathrm{~m} \leq \lambda \leq 310^{-5} \mathrm{~m}$, they are stronger than the previously known ones by about $10^{3}$ times and near $\lambda \approx 610^{-6}$, they are $310^{3}$ times stronger. The corresponding constraints which result for the masses of the spin-one antigraviton and for the dilaton are also discussed.

\section{Calculation of the Hypothetical Forces for the Configuration Used in the Experiment}

In the recent experiment [10] the Casimir force was measured between a plane disc of diameter $d=2.54 \mathrm{~cm}$ and thickness $D=0.5 \mathrm{~cm}$ and a lens of height $H=0.18 \mathrm{~cm}$, diameter $L=4 \mathrm{~cm}$ and curvature radius $R=11.3 \mathrm{~cm}$. The surfaces of the plate and the lens were covered with copper and gold layers of $0.5 \mu \mathrm{m}$ thickness each (see 
Fig. 1). For this configuration the Casimir force is attractive and given by [11]

$$
F=-\frac{2 \pi R}{3} \frac{\pi^{2}}{240} \frac{\hbar \mathrm{c}}{a^{3}}
$$

This expression was later obtained by another method in Ref. [13] (see also Refs. [6, 14]). In fact it is an approximation but, as shown in [6, 14], for a large radiusof-curvature lens, the relative error is less than $0.01 \%$.

The potential of the hypothetical interaction between the atoms of the disc and the atoms in the lens has the form

$$
V(r)=-\alpha \hbar \mathrm{c} N_{1} N_{2} \frac{\exp (-r / \lambda)}{r}
$$

where $r$ is the interatomic distance, $\alpha$ is the (dimensionless) interaction constant, $\lambda=$ $\hbar / \mathrm{mc}$ is the range of the interaction and simultaneously the Compton wavelength of the hypothetical particles whose exchange leads to the hypothetical forces under discussion. The coefficients $N_{1}, N_{2}$ are the numbers of nucleons in the atoms so that the constant $\alpha$ is independent of the type of atoms, i.e., on the nuclear charges.

Due to its smallness the hypothetical interaction between solid bodies can be obtained by summing the potential (2) over their volumes

$$
\begin{aligned}
V(a)= & -\alpha \frac{\hbar \mathrm{c}}{m_{\mathrm{p}}^{2}}\left[\rho_{1} \int_{V_{1}} \mathrm{~d}^{3} \mathbf{r}_{1}+\rho_{2} \int_{V_{2}} \mathrm{~d}^{3} \mathbf{r}_{1}+\rho_{3} \int_{V_{3}} \mathrm{~d}^{3} \mathbf{r}_{1}\right] \\
& \times\left[\tilde{\rho}_{1} \int_{\tilde{V}_{1}} \mathrm{~d}^{3} \mathbf{r}_{2}+\rho_{2} \int_{\tilde{V}_{2}} \mathrm{~d}^{3} \mathbf{r}_{2}+\rho_{3} \int_{\tilde{V}_{3}} \mathrm{~d}^{3} \mathbf{r}_{2}\right] \frac{\exp (-r / \lambda)}{r},
\end{aligned}
$$

where $r=\left|\mathbf{r}_{1}-\mathbf{r}_{2}\right|$. The vectors $\mathbf{r}_{1}$ and $\mathbf{r}_{2}$ belong to the volumes indicated in the lower integration limits in the operator brackets. This formula reflects the composition of the disc and the lens, both having metal layers, used in the experiment. In (3), by means of $\rho=n N m_{\mathrm{p}}$, where $m_{\mathrm{P}}$ is the proton mass, we introduced the densities of the materials used in the experiment: $\rho_{1}$ and $\tilde{\rho_{1}}$ for the quartz in the disc and the lens, respectively and $\rho_{2,3}$ for the copper and gold layers $(n$ being the atomic densities).

Using this formula, the hypothetical force between the disc and the lens can be expressed as

$$
F_{H}=-\frac{\partial V(a)}{\partial a}
$$

The integrals in these expressions cannot be taken explicitely. In general, numerical integration is required (see below). However, if the part of the lens, which contributes most to the result is small compared with the disc, the integrals in (3) can easily be taken explicitely. This approximation is in any case justified for $\lambda \leq 10 \mu \mathrm{m}$. In fact, its validity can be extended up to $\lambda_{\sim}^{<} 100 \mu \mathrm{m}$ as the numerical calculations show.

In order to perform the calculation for an infinite disc we first consider the potential energy of one lens atom having $N_{L}$ nucleons in its nucleus at position 
$(0,0, l)$ above the disc so that the distance to an atom within the disc at position $(x, y, z)$ is $r=\sqrt{x^{2}+y^{2}+(z-l)^{2}}$ (see Fig. 1)

$$
\begin{gathered}
V_{A}(l)=-\alpha N_{L} \frac{\hbar \mathrm{c}}{m_{\mathrm{p}}} \int_{-\infty}^{\infty} \mathrm{d} x \int_{-\infty}^{\infty} \mathrm{d} y\left[\rho_{1} \int_{-D}^{-2 \Delta} \mathrm{d} z+\rho_{2} \int_{-2 \Delta}^{-\Delta} \mathrm{d} z\right. \\
\left.+\rho_{3} \int_{-\Delta}^{0} \mathrm{~d} z\right] \frac{\exp (-r / \lambda)}{r}
\end{gathered}
$$

Performing the integration in (5) we get

$$
V_{A}(l)=K N_{L} \exp (-l / \lambda)
$$

where the coefficient $K$ is given by

$$
\begin{aligned}
K= & -2 \pi \alpha \lambda^{2} \frac{\hbar \mathrm{c}}{m_{\mathrm{p}}}\left[\left(\rho_{1}-\rho_{2}\right) \exp (-2 \Delta / \lambda)\right. \\
& \left.+\left(\rho_{2}-\rho_{3}\right) \exp (-\Delta / \lambda)-\rho_{1} \exp (-D / \lambda)+\rho_{3}\right] .
\end{aligned}
$$

In order to get the complete interaction potential it is necessary to integrate (6) over the volume of the lens, multiplying by the corresponding atomic densities at height $l$. We obtain

$$
\begin{aligned}
V(a)=\frac{K}{m_{\mathrm{p}}}\left\{\int_{a+2 \Delta}^{a+H}\left[\gamma_{1}(l)+\gamma_{2}^{(1)}(l)+\gamma_{3}^{(1)}(l)\right] \exp (-l / \lambda) \mathrm{d} l\right. \\
\\
+\int_{a+\Delta}^{a+2 \Delta}\left[\gamma_{2}^{(2)}(l)+\gamma_{3}^{(1)}(l)\right] \exp (-l / \lambda) \mathrm{d} l \\
\left.+\int_{a}^{a+\Delta} \gamma_{3}^{(2)}(l) \exp (-l / \lambda) \mathrm{d} l\right\},
\end{aligned}
$$

where the $\gamma$ 's are in fact proportional to the areas of sections of the lens at height $l$ over the disc. They are given by:

$$
\gamma_{1}(l)=\pi \tilde{\rho_{1}}\left[2 R(l-a-2 \Delta)-(l-a)^{2}+4 \Delta^{2}\right]
$$

(for the quartz atoms within $a+2 \Delta \leq l \leq a+H$ ),

$$
\gamma_{2}^{(1)}(l)=\pi \rho_{2} \Delta(2 R-3 \Delta)
$$

(for the copper atoms within $a+2 \Delta \leq l \leq a+H$ ),

$$
\gamma_{3}^{(1)}(l)=\pi \rho_{3} \Delta(2 R-\Delta)
$$

(for the gold atoms within $a+\Delta \leq l \leq a+H$ ),

$$
\gamma_{2}^{(2)}(l)=\pi \rho_{2}\left[2 R(l-a-\Delta)-(l-a)^{2}+\Delta^{2}\right]
$$


(for the copper atoms within $a+\Delta \leq l \leq a+2 \Delta$ ),

$$
\gamma_{3}^{(2)}(l)=\pi \rho_{3}\left[2 R(l-a)-(l-a)^{2}\right]
$$

(for the gold atoms within $a \leq l \leq a+\Delta$ ).

Performing the integration in (8) we obtain the final expression for the force $F_{H}^{\text {inf }}$ between an infinite disc and the lens:

$$
\begin{aligned}
F_{H}^{i n f}= & -2 \pi^{2} \alpha \frac{\lambda^{2} \hbar \mathrm{c}}{m_{\mathrm{p}}^{2}} \mathrm{e}^{-a / \lambda}\left[\rho_{3}-\left(\rho_{2}-\rho_{1}\right) \mathrm{e}^{-2 \Delta / \lambda}-\left(\rho_{3}-\rho_{2}\right) \mathrm{e}^{-\Delta / \lambda}-\rho_{1} \mathrm{e}^{-D / \lambda}\right] \\
& \times\left\{2 \lambda \rho_{3}(R-\lambda)-2 \lambda\left(\rho_{3}-\rho_{2}\right)(R-\Delta-\lambda) \mathrm{e}^{-\Delta / \lambda}\right. \\
& -2 \lambda\left(\rho_{2}-\tilde{\rho_{1}}\right)(R-2 \Delta-\lambda) \mathrm{e}^{-2 \Delta / \lambda} \\
& +\left[2 R \lambda \tilde{\rho_{1}}\left(-1+\lambda / R-H / \lambda+H / R+H^{2} / 2 R \lambda\right)\right. \\
& \left.\left.+\Delta^{2}\left(\rho_{3}+3 \rho_{2}-4 \tilde{\rho_{1}}\right)-2 R \Delta\left(\rho_{2}+\rho_{3}-2 \tilde{\rho_{1}}\right)\right] \mathrm{e}^{-H / \lambda}\right\} .
\end{aligned}
$$

\section{Constraints for the Parameters of the Yukawa- Type Interaction}

The expression (1) for the Casimir force acting between a flat plate and a spherical lens was confirmed experimentally in [10] for a range of distance $0.6 \mu \mathrm{m} \leq a \leq 6 \mu \mathrm{m}$ with a relative error of $\delta=5 \%$. This means that the value of the hypothetical forces cannot surpass five percents of the Casimir force. Consequently the inequality

$$
\left|F_{H}\right| \leq 0.05|F|
$$

where $F_{H}$ is given by Eqs. (3), (4) or, in the case of sufficiently small $\lambda$ by Eq. (14), holds.

From the inequality (15) the constraints on the parameters $\alpha$ and $\lambda$ of the hypothetical force $F_{H}$ follow. We consider this in what follows. Note that for the range $\lambda \leq 10^{-7} \mathrm{~m}$ the force is well approximated by the simple formula following from (14)

$$
F_{H}^{i n f}=-4 \pi^{2} \alpha \frac{\lambda^{3} \hbar \mathrm{c} R}{m_{\mathrm{p}}^{2}} \rho_{3}^{2} \mathrm{e}^{-a / \lambda} .
$$

Using (11), (16), the inequality (15) may be written in the form

$$
|\alpha| \leq \frac{5 \pi}{1.4410^{5}} \frac{m_{\mathrm{p}}^{2}}{\rho_{3}^{2} \lambda^{3}} \frac{1}{a^{3}} \mathrm{e}^{a / \lambda} .
$$

It is seen from (17) that the strength of the constraint in this range does not depend on $R$, the radius of the lens.

For the range $10^{-8} \mathrm{~m} \leq \lambda \leq 1.610^{-4} \mathrm{~m}$ the constraints are obtained using formula (14). For the range $1.610^{-4} \mathrm{~m} \leq \lambda \leq 10^{2} \mathrm{~m}$ it is impossible to consider the radius 
of the disc as large and a numerical integration in formula (3) was performed. For this purpose algorithm 698 from netlib (http://www.netlib.org) was used. It is an adaptive multidimensional integration, the Fortran program is called 'dcuhre'. A large number of function calls (about $510^{6}$ ) for $\lambda \geq 10^{-4} \mathrm{~m}$ was necessary in order to obtain reliable numerical values. For smaller $\lambda$ the program does not work, but there the radius of the disc becomes unimportant and formula (14) can be used. Note that, e.g., for $\lambda=10^{-3} \mathrm{~m}$ the hypothetical force acting between the lens and the finite disc is $72.3 \%$ of the force for an infinite disc. For $\lambda=10^{-4} \mathrm{~m}$ it is already $99.96 \%$.

The values of the geometrical parameters used in [10 are given in Sec. 2. The densities of the materials of the disc and of the lens are $\rho_{1}=2.4 \mathrm{~g} / \mathrm{cm}^{3}$ for the quartz in the lens and $\tilde{\rho}_{1}=2.23 \mathrm{~g} / \mathrm{cm}^{3}$ for the quartz in the disc, $\rho_{2}=8.96 \mathrm{~g} / \mathrm{cm}^{3}$ and $\rho_{3}=19.32 \mathrm{~g} / \mathrm{cm}^{3}$ for copper and gold, correspondingly.

The results of all calculations are collected into inequality (15) in order to obtain the new constraints on the constants of hypothetical long-range interactions. The obtained constraints are shown by curve 1 in Fig. 2, where the region above the curve is ruled out by the cited experimental data. Thereby for every value of $\lambda$ the value of $a$ was used which gives the best constraint. This is $a=6 \mu \mathrm{m}$ for large $\lambda$ and $a=0.6 \mu \mathrm{m}$ for small $\lambda$ and correspondingly in between. For comparison, in the same Fig. 2 the curve 2 shows the previously known [5, 6, 8] constraints for the Yukawa-type hypothetical interactions which follow from the experiment [11] on Casimir force measuring. In that experiment the lens and the disc (it was large as compared with the lens) were made out from quartz only (without metallic layers on the surfaces). The dependence (1) with an appropriate correction factor due to the dielectricity was confirmed with a relative error of $\delta=10 \%$ for distances $0.1 \mu \mathrm{m} \leq a \leq 1 \mu \mathrm{m}$. As a result, for extremely small $\lambda \approx 10^{-8} \mathrm{~m}$ the experiment [11] gave stronger constraints than the experiment [10]. This constraint results from the smallest values $a=0.1 \mu \mathrm{m}$. This becomes clear when taking into account that the function $a^{-3} \exp (a / \lambda)$ appearing in (17) takes its minimal value for $a=3 \lambda$. Therefore the experiment [11] with the smallest value of $a$ gives the best results in this case.

For $\lambda \geq 6.310^{-8} \mathrm{~m}$, as can be seen from Fig. 2, the experiment under consideration gives much stronger constraints than that of [11]. In the range $210^{-6} \mathrm{~m} \leq \lambda \leq$ $310^{-5} \mathrm{~m}$ the new constraints surpass the old ones by more than a factor of 1000 and near $\lambda=610^{-6}$, by 3000. For $\lambda \approx 10^{-4} \mathrm{~m}$, where the old Casimir experiment has given the same constraints as one of the Cavendish-type experiments, the increase is 400 times.

The constraints which follow from the Cavendish- and Eötvös-type experiments are also shown in Fig. 2 [5, 6, 8]. Curve 3 follows from the experiment [15], curve 4 from [16] and curve 5 from [17], curve 6 from the Eötvös-type experiment [18]. As shown in Fig. 2, in the range $10^{-4} \mathrm{~m} \leq \lambda \leq 3.110^{-3} \mathrm{~m}$ the new constraints from the Casimir effect surpass in some points the results from the Cavendish-type experiment in Ref. [14], in some points they are of the same strength (compare the curves 1 and 3 in Fig. 2). 
The new constraints resulting from the recent Casimir force measurement (in distinction from the old ones) give the possibility to get constraints for the masses of the spin-one antigraviton and the dilaton. The exchange of spin-one antigravitons gives rise to repulsive forces described by (1) with $\alpha_{a}=-8 \pi \mathrm{Gm}_{\mathrm{qe}}^{2} /(\hbar \mathrm{c})$, where $\mathrm{m}_{\mathrm{qe}} \approx 365 \mathrm{MeV}$ is the sum of the masses of the current quarks belonging to a nucleus and of atomic electrons, and $\mathrm{G}$ is the gravitational constant. This gives $\alpha \approx 10^{-40}$ and from curve 1 of Fig. 2 it follows that $\lambda_{a} \leq 510^{-3} \mathrm{~m}$, or for the spinone antigraviton mass, $m_{a} \geq 3.610^{-5} \mathrm{eV}$. This constraint is only slightly weaker than the previous one $\left(\lambda_{a} \leq 310^{-3} \mathrm{~m}, m_{a} \geq 610^{-5} \mathrm{eV}\right.$, [5]) obtained from the Cavendish-type experiment of Ref. [16] (shown as curve 4 in Fig. 2).

A similar constraint follows for the dilaton whose interaction constant is given by theory to be $\alpha_{d}=\frac{1}{3} G m_{\mathrm{p}}^{2} /(\hbar \mathrm{c}) \approx 210^{-39}$. As seen from Fig. 2, for such a constant $\lambda_{d} \leq 5.510^{-4} \mathrm{~m}$ or, in terms of the dilaton mass, $m_{d} \geq 3.310^{-4} \mathrm{eV}$ follows. Quite the same constraint follows from the Cavendish-type experiment of Ref. [15] (shown as curve 3 in Fig. 2). Therefore in the region $10^{-4} \mathrm{~m} \leq \lambda_{d} \leq 3.110^{-3} \mathrm{~m}$ the constraints following from the Casimir effect are approximately of the same strength as those which follow from Cavendish-type experiments.

\section{Conclusion and Discussion}

As discussed in the preceeding sections, the new measurement of the Casimir force presented in [10] makes it possibile to strengthen the constraints of the constants of hypothetical long-range interactions by a factor of 1000 and more for a wide interaction range. Moreover, it it is also possible to widen (by one and a half orders of magnitude) the boundary of the $\lambda$-interval for which the Casimir effect gives good constraints for Yukawa-type hypothetical interactions. In this way the Casimir effect becomes a strong competitor to the Cavendish-type experiments in obtaining constraints for such interactions.

To obtain even stronger constraints from the Casimir effect it would be desirable to enlarge the distance interval over which the force is measured, perhaps up to $0.1 \mu \mathrm{m} \leq a \leq 10 \mu \mathrm{m}$. Near the left boundary of this interval, one must take into account the contributions to the Casimir force resulting from the uneveness and imperfections of the disc and the lens [14]. Near the right boundary, the contributions due to the temperature corrections [6] should be considered too. Also, the use of materials with high density for the whole disc and lens would be helpful. Finally, a further reduction of the error in the force measurement would be important.

\section{Acknowledgments}

The authors are greatly indebted to S.K. Lamoreaux for sending the manuscript of his paper [10] before publication and for several helpful discussions, in particular for communicating the densities $\rho_{1}$ and $\tilde{\rho_{1}}$. They also thank A.J. Sanders for his interest in this work and for facilitating communication. 
One of us (V.M.M.) is indebted to the Institute for Theoretical Physics of the University of Leipzig, where this work was partly performed, for kind hospitality and to the DFG under grant 436 RUS 17/88/94 for support. 


\section{References}

[1] J. Kim, Phys. Rep. C 150, 1 (1987).

[2] E.G. Adelberger et al, Annu. Rev. Nucl. Sci. 41, 269 (1991).

[3] G.T. Gillies, Metrologia Suppl. 24, 1 (1987).

[4] E. Fischbach et al, Metrologia 29, 215 (1992).

[5] V.M. Mostepanenko and I.Yu. Sokolov, Phys. Rev. D 47, 2882 (1993).

[6] V.M. Mostepanenko and N.N. Trunov, The Casimir Effect and its Applications. Clarendon Press, Oxford, 1997.

[7] V.A. Kuz'min, I.I. Tkachev and M.E. Shaposhnikov, JETP Letters (USA) 36, 59 (1982).

[8] V.M. Mostepanenko, I.Yu. Sokolov, Phys. Lett. A132, 313 (1988).

[9] M. Bordag, V.M. Mostepanenko and I.Yu. Sokolov, Phys. Lett. A187, 35 (1994).

[10] S.K. Lamoreaux, Phys. Rev. Lett. 78, 5 (1997).

[11] B.V. Derjaguin, I.I. Abrikosova and E.M. Lifshitz, Quart. Rev. 10, 295 (1968).

[12] M.J. Sparnaay, Physica 24, 751 (1958).

[13] V.M. Mostepanenko and I.Yu. Sokolov, Sov. Phys. Dokl. (USA) 33, 140 (1988).

[14] G.L. Klimchitskaya and Yu.V. Pavlov, Int. J. Mod. Phys. A11, 3723 (1996).

[15] V.P. Mitrofanov and O.I. Ponomareva, Sov. Phys. JETP 67, 1963 (1988).

[16] J.K. Hoskins et al, Phys. Rev. D 32, 3084 (1985).

[17] Y.T. Chen, A.H. Cook and A.J.F. Metherell, Proc. Roy. Soc. Lond. A394, 47 (1984).

[18] B.R. Heckel et al, Phys. Rev. Lett. 63, 2705 (1989). 


\section{List of Captions}

Fig. 1 The configuration of a flat disc with diameter $d$ and thickness $D$, and a spherical lens with curvature radius $R$, height $h$ and diameter $L$ used in the experimental measurement of the Casimir force [10]. The volumes $V_{1}$ and $\tilde{V}_{1}$ are occupied by quartz, $V_{2}, \tilde{V}_{2}$ and $V_{3}, \tilde{V}_{3}$ are the copper and gold layers of thickness $\Delta$ each.

Fig. 2 Constraints for the constants of hypothetical Yukawa-type interactions following from the new measurement of the Casimir force [10 (curve 1), from the old Casimir force measurements between dielelectric bodies [11 (curve 2), from the Cavendish-type experiments 15 (curve 3), Ref. 16 (curve 4), Ref. [17 (curve 5) and from Eötvös-type experiments [18] (curve 6). The permitted regions of $\alpha$ and $\lambda$ lie below the curves. 


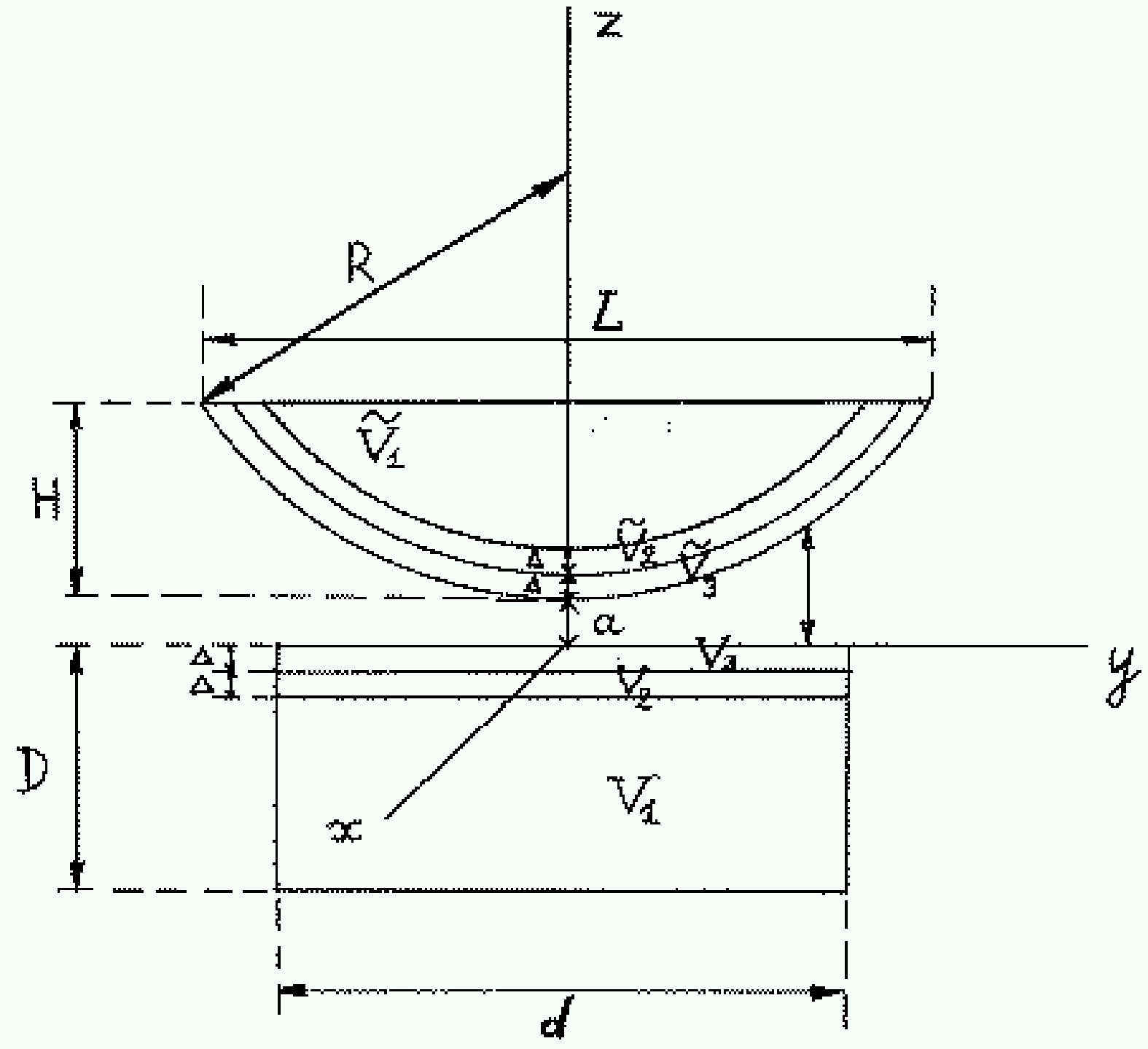

Figure 1 


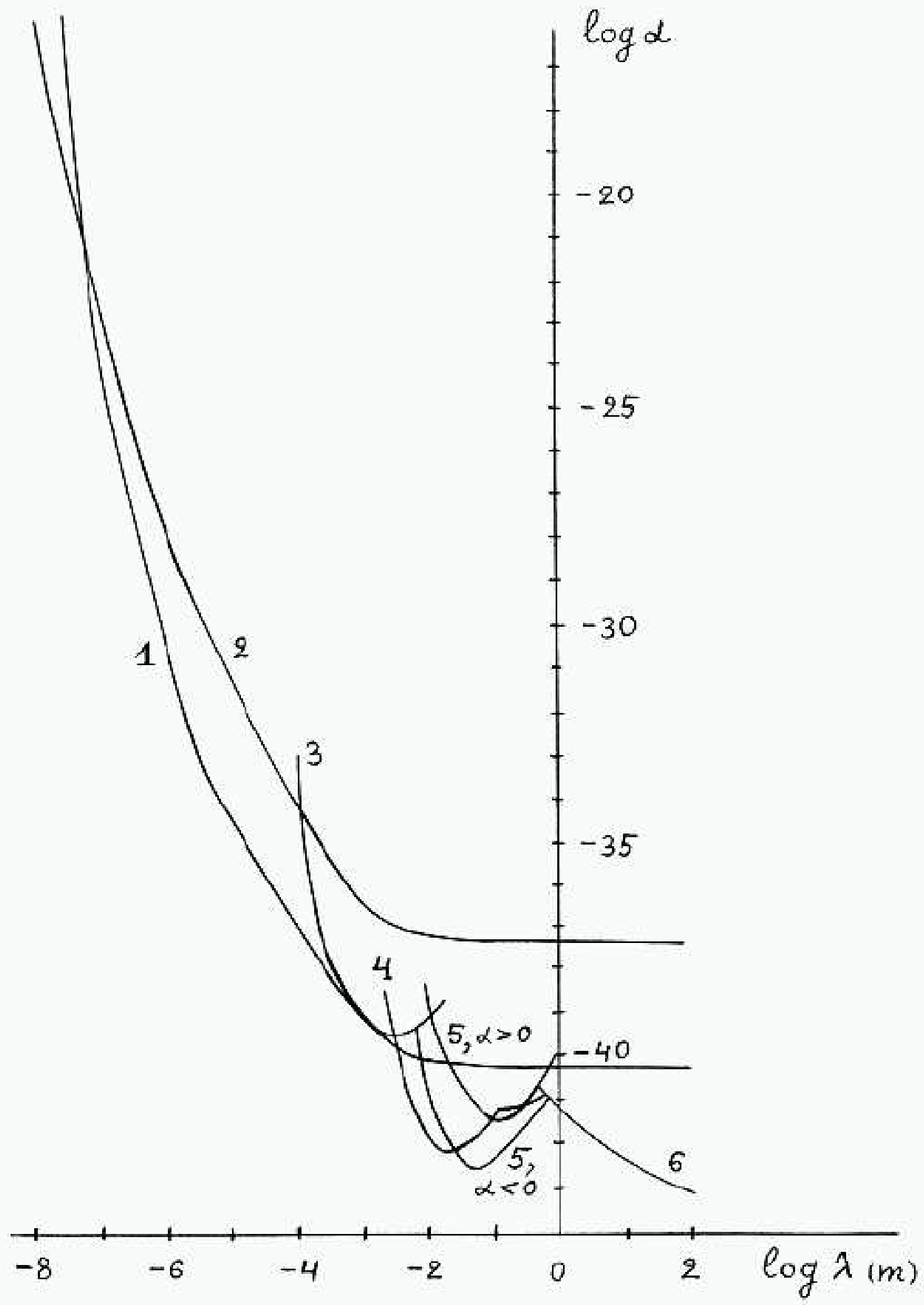

Figure 2 